\title{
Effects of aging and annealing on the polar and antiferrodistortive components of the antiferroelectric transition in $\mathrm{PbZr}_{1-x} \mathrm{Ti}_{x} \mathrm{O}_{3}$
}

\author{
F. Cordero, ${ }^{1}$ F. Trequattrini, ${ }^{2}$ F. Craciun, ${ }^{1}$ C. Galassi ${ }^{3}$ \\ 1 CNR-ISC, Istituto dei Sistemi Complessi, Area della Ricerca di Roma - Tor Vergata, \\ Via del Fosso del Cavaliere 100, I-00133 Roma, Italy \\ 2 Dipartimento di Fisica, Università di Roma "La Sapienza", P.le A. Moro 2, I-00185 Roma, Italy and \\ ${ }^{3}$ CNR-ISTEC, Istituto di Scienza e Tecnologia dei Materiali Ceramici, Via Granarolo 64, I-48018 Faenza, Italy
}

(Dated:)

\begin{abstract}
The antipolar and antiferrodistortive (AFD) components of the antiferroelectric (AFE) transition in $\mathrm{PbZr}_{1-x} \mathrm{Ti}_{x} \mathrm{O}_{3}(x \leq 0.054)$ can occur separately and with different kinetics, depending on the sample history, and are accompanied by elastic softening and stiffening, respectively. Together with the softening that accompanies octahedral tilting in the fraction of phase that is not yet transformed into AFE, they give rise to a variety of shapes of the curves of the elastic compliance versus temperature. All such anomalies found in samples with $x=0.046$ and 0.054 , in addition to those already studied at $x=0.050$, can be fitted consistently with a phenomenological model based on the simple hypothesis that each of the polar and AFD transitions produces a step in the elastic modulus, whose position in temperature and width reflect the progress of each transition. The slowing of the kinetics of the transformations is correlated with the formation of defect structures during aging in the ferroelectric (FE) or AFE state, which are also responsible for a progressive softening of the lattice with time and thermal cycling, until annealing at high temperature recovers the initial conditions.
\end{abstract}

\section{INTRODUCTION}

The AFE/FE transition in $\mathrm{PbZrO}_{3}$ based perovskites involves large volume changes and therefore is widely studied in view of applications for actuators, $\frac{1}{-1}$ but also other applications are envisaged, such as high energy storage capacitors or cooling devices $\stackrel{2}{2}$ The large strains involved in the transition may be partly responsible for the slow kinetics of the FE to AFE transition driven by cooling, and hence large thermal hysteresis, and may even cause lattice damage ranging from broken bonds ${ }^{3,4}$ to cracks. .6 The slow kinetics of AFE/FE transitions has been the object of experimental and theoreti$\mathrm{cal} /$ phenomenological modeling, including a series of articles by Ishchuk and coworkers, $\underset{7}{7}$ who propose that short range cation migration is involved, and is made possible at room temperature by the large stress at the $\mathrm{AFE} / \mathrm{FE}$ interfaces .8 The physics of the AFE/FE transition can be particularly rich, and these authors even suggest that the relaxor transition can be explained in terms of coexisting FE and AFE phases .9

An additional phenomenon often related to the AFE transitions is the existence of incommensurate structural modulations. Local structural probes like electron diffraction reveal that the AFE transition may be preceded by incommensurate modulations of the cation displacements, and similar coexistence of incommensurate modulated AFE structures with the paraelectric or FE phase have been observed in $\mathrm{Pb}\left(\mathrm{Sc}_{1 / 2} \mathrm{Ta}_{1 / 2}\right) \mathrm{O}_{3},{ }^{10}$ and other perovskites, including variously doped ${ }^{11}$ but also undoped ${ }^{12} \mathrm{PbZr}_{1-x} \mathrm{Ti}_{x} \mathrm{O}_{3}$ (PZT).

A new type of information came from the combination of elastic, dielectric and diffraction experiments on PZT, 13 that showed how the polar and antiferrodistortive (AFD) degrees of freedom can act almost inde- pendently of each other and with very different kinetics in the $\mathrm{AFE} / \mathrm{FE}$ transition. In fact, the transition from the rhombohedral (R) FE phase to the orthorhombic $(\mathrm{O})$ AFE phase involves both cation displacements and AFD tilting of the $\mathrm{O}$ octahedra according to the $a^{-} a^{-} c^{0}$ pattern, in Glazer's notation. ${ }^{14}$ While the FE and AFE cation modes are easily detectable by diffraction and produce large effects in the dielectric response, the transitions involving AFD modes produce clear steps in the elastic moduli, which are also sensitive to the polar transitions. We showed that in PZT with 5\% Ti the $\mathrm{R}-\mathrm{FE} / \mathrm{O}-\mathrm{AFE}$ transition involves the concomitant condensation or ordering of both AFD and polar modes in quasistatic experiments, but the AFE component could be much slower or even absent when cooling at rates above $0.5 \mathrm{~K} / \mathrm{min}^{13}$ The curves of the Young's modulus as a function of temperature were found to reflect the progress of the various polar and AFD transitions, and exhibited a variety of shapes, depending on the sample history and temperature rate.

Here we extend the experiments to compositions of $4.6 \%$ and $5.4 \% \mathrm{Ti}$ and find that it is also possible that the AFD transition is slower than the AFE one. Similarly to the previous work, 13 to which we refer for the region of interest of the phase diagram of $\mathrm{PZT}$, the variety of shapes of elastic anomalies found under different conditions can be fitted setting the compliance as the volume average of the compliances of the four possible phases coexisting in that region of the phase diagram, counting the $a^{-} a^{-} c^{0}$ tilt pattern and the AFE cation ordering as separate phases, and including the tilted R-FE phase $R 3 c$. Here we added the option of imposing that the $\mathrm{O}$ tilt pattern sets in only in the AFE phase. The slow kinetics of the transformations are correlated with the formation of defect structures during aging in the FE 
or AFE state, which are also responsible for a progressive softening of the lattice with time and thermal cycling.

It may be interesting to note that in multiferroic $\mathrm{BiFeO}_{3}$ there is a nanoscale alternation of R-FE $R 3 c$ and $\mathrm{O}-\mathrm{AFE}$ domains with the same tilt patterns as in PZT (but with different AFE pattern of the Bi atoms) $\stackrel{15}{\stackrel{P}{5} \text { and }}$ a modulated superstructure involving tilting and AFE displacements of $\mathrm{Ti}$ is found in $\mathrm{EuTiO}_{3}$, another multiferroic perovskite ${ }^{16}$ Likely, understanding the mechanisms of interaction between the tilt and polar modes in AFE PZT provides insight into those operating in the multiferroic perovskites and viceversa.

\section{EXPERIMENTAL}

Ceramic samples of $\mathrm{PbZr}_{1-x} \mathrm{Ti}_{x} \mathrm{O}_{3}$ (abbreviated as PZT100x) with $x=0.046$ and 0.054 were prepared in the same manner as previous series of samples $\frac{17,18}{12}$ The oxide powders were calcined at $800{ }^{\circ} \mathrm{C}$ for 4 hours, pressed into bars, sintered at $1250{ }^{\circ} \mathrm{C}$ for $2 \mathrm{~h}$ in crucibles packed with $\mathrm{PbZrO}_{3}+5$ wt\% excess $\mathrm{ZrO}_{2}$ to prevent $\mathrm{PbO}$ loss. The powder X-ray diffraction did not reveal any trace of impurity phases. The densities were about $95 \%$ of the theoretical values and the grains were large, with sizes of $5-20 \mu \mathrm{m}$. The bars were $4 \mathrm{~cm}$ long and cut into slices $0.6 \mathrm{~mm}$ thick. In order to measure the dynamic Young's modulus $E(\omega)=E^{\prime}-i E^{\prime \prime}$, the sample was suspended on thin thermocouple wires in vacuum and electrostatically excited on its first odd flexural modes with an electrode close to its surface, partially made conducting with Ag paint. Sample and electrode also constitute a capacitor inserted in an oscillator whose high-frequency of $\sim 150 \mathrm{kHz}$ is modulated by the sample vibration and can be demodulated to produce a signal proportional to the sample vibration $\frac{19}{19}$ Below we present the reciprocal of the Young's modulus, the compliance $s(\omega, T)=s^{\prime}-i s^{\prime \prime}$, which is the mechanical analogue of the dielectric susceptibility, measured on the fundamental mode with $\omega / 2 \pi$ $\sim 1.6 \mathrm{kHz}$. Since $\omega \propto \sqrt{E^{\prime}}, 20$ the temperature variation of $s$ is given by $s(T) / s_{0} \simeq \omega_{0}^{2} / \omega^{2}(T)$, where $\omega_{0}$ is chosen so that $s_{0}$ is the compliance in the paraelectric phase. The elastic losses are presented as $s^{\prime \prime} / s^{\prime}=Q^{-1}$, where the mechanical $Q$ of the sample was measured from the width of the resonance curve or from the decay of the free oscillations after switching off the excitation.

The dielectric permittivity $\varepsilon(\omega, T)=\varepsilon^{\prime}-i \varepsilon^{\prime \prime}$ was measured with a HP $4284 \mathrm{~A}$ LCR meter with a four-wire probe and an electric field of $0.5 \mathrm{~V} / \mathrm{mm}$, between 0.2 and $200 \mathrm{kHz}$ on disc samples with $12 \mathrm{~mm}$ diameter and $0.7 \mathrm{~mm}$ thick. Temperature was controlled with a modified Linkam HFS600E-PB4 stage.

\section{RESULTS AND DISCUSSION}

The same notation as in the previous study on PZT5 will be adopted. We do not attribute space groups to the intermediate phases undergoing the partial AFE or AFD transformations for two reasons. One is that the dielectric and elastic susceptibilities do not provide information on the cell structure, but only on the type of the transformation. The other reason is that, as discussed in Sect. IIIE even the starting R-FE phase for $x(\mathrm{Ti})<0.06$ has an average $R 3 m$ structure, but the octahedra should be already tilted below $T_{\mathrm{C}}$, though without long range order $\stackrel{18,21}{ }$ Therefore, already the description of the starting $\mathrm{FE}$ phase might require two symmetry groups: one for the average structure determined by neutron and X-ray diffraction, and lower symmetries for the local structure. The structure within the broad temperature region of the transformation between the FE $R 3 m$ and AFE Pbam would require an even more complex description, which we simply denote as $\sim$ Pbam.

The nomenclature of the various transitions is detailed in Table I, and the following abbreviations will be used: $\mathrm{AF}$ indicates the polar component of the AFE/FE transition; OT (= octahedral tilting $a^{-} a^{-} c^{0}$ ) the AFD component; $\mathrm{T}$ the tilting transition within the FE-R phase between untilted $R 3 m$ and $a^{-} a^{-} a^{-}$tilted $R 3 c$. The transition temperatures will be identified with the centers of the steps of $s^{\prime}(T)$ and called $T_{k}^{h / c}$ where $k=\mathrm{AF}, \mathrm{OT}, \mathrm{T}$ and $h / c$ specify heating/cooling.

\section{A. Characters of the transformations}

Both the FE/PE transition at $T_{\mathrm{C}}$ and the AFD at $T_{\mathrm{T}}$ involve a single order parameter (OP) and can in principle be of the second order. Indeed, they exhibit little hysteresis between heating and cooling. Instead, the transition between the FE $R 3 m$ or $R 3 c$ and the AFE Pbam phases must be first order and involves two separate OPs. The polar mode passes from predominant displacements of Ti along (111) (pseudocubic setting) in the FE phase to predominant displacements of $\mathrm{Pb}$ along $\langle 1 \overline{1} 0\rangle$ with wave vector $\left(\frac{1}{4} \frac{1}{4} 0\right)$ in the AFE phase, without group-subgroup relationship between the two phases. ${ }^{22,23}$ An additional AFD order parameter is responsible for the $a^{-} a^{-} c^{0}$ tilt pattern. In pure $\mathrm{PbZrO}_{3}$ the transition is ideally from the cubic phase, and the temperature dependence of the two OPs has been described within the Landau formalism in terms of a primary polar OP and a secondary AFD OP bilinearly coupled with the former and nearly following it. 24

The situation with 5\% Ti doping has been shown to be quite different,$\stackrel{13}{ }$ not only because the parent phase is R-FE instead of cubic PE, but also because the AFD component of the transition can occur while the antipolar one is frozen or hindered, suggesting exchanged roles of the two OPs. Yet, at the two compositions studied here, very close to $5 \% \mathrm{Ti}$, also the reverse is true: the AFE OP may advance the AFD one. Evidently, at these compositions near the morphotropic phase boundary with the FE phase, the free energy is rather flat between the FE and AFE minima, and also with respect to various tilt 
TABLE I: Transition temperatures with meaning and phases that they separate. Superscript $\mathrm{h} / \mathrm{c}=\mathrm{heating} / \mathrm{cooling}$; L/SRO $=$ long/short range order. It is assumed that for $<6 \% \mathrm{Ti}$ it is $T_{\mathrm{IT}}=T_{\mathrm{C}}$ and therefore the $R 3 \mathrm{~m}$ phase is tilted with $\mathrm{SRO}{ }^{18,21}$

\begin{tabular}{lll}
\hline \hline$T_{\mathrm{C}}$ & Curie temperature $(\mathrm{FE} \leftrightarrow \mathrm{PE})$ & $R 3 m \leftrightarrow P m 3 m$ \\
$T_{\mathrm{T}}$ & tilting (LRO $\left.a^{-} a^{-} a^{-}\right)$ & $R 3 c \leftrightarrow R 3 m$ (untilted or SRO tilted) \\
$T_{\mathrm{AF}}$ & $\mathrm{AFE} \leftrightarrow \mathrm{FE}($ cation displacements) & $\sim P b a m \leftrightarrow R 3 m$ \\
$T_{\mathrm{OT}}$ & orthorhombic tilt $\left(\mathrm{LRO} a^{-} a^{-} c^{0}\right)$ & $\sim P b a m \leftrightarrow R 3 m$ \\
$T_{\mathrm{IT}}$ & intermediate tilt $=\mathrm{SRO}$ tilting & $R 3 m$ SRO tilted $\leftrightarrow R 3 m$ untilted \\
\hline \hline
\end{tabular}

patterns, so that these minima can be easily perturbed by slight changes in $\mathrm{Zr} / \mathrm{Ti}$ distributions, extrinsic defects and possibly even by twin and domain walls. This results in a readiness to change the balance and kinetics of the two OPs upon small changes in the sample preparation and history, and renders the description of the evolution of the AFE phase transition much less transparent than for pure or lightly doped $\mathrm{PbZrO}_{3}{ }^{24}$

As mentioned in the Introduction and in the previous study, 13 observations of exceptionally large thermal hystereses and slow kinetics of the AFE transition in undoped and variously doped PZT have been done with various techniques. A large thermal hysteresis and coexistence of $R 3 \mathrm{~m}, R 3 c$ and Pbam phases result from dielectric and pyroelectric measurements on PZT with $x(\mathrm{Ti})=0.05^{25}$ and with Raman scattering in Nb-doped PZT with $x \leq 0.03, \stackrel{26}{n}$ while TEM observations ${ }^{27,28}$ provide additional information on the local octahedral tilting, and are consistent with the idea that the $R 3 \mathrm{~m}$ phase at low $x$ is already tilted, though with a disordered or incommensurate pattern. The nucleation of the AFE phase has been followed also directly with a polarized microscope, ${ }^{29}$ finding that its athermal character in pure $\mathrm{PbZrO}_{3}$, namely independent of thermal fluctuations and hence unchanged under stationary conditions, is changed by the Ti substitution into strongly isothermal, where the evolution can slowly proceed at constant temperature. 29 Codoping with $\mathrm{La}$ and $\mathrm{Li}$ on the $\mathrm{Pb}$ site adds new possible mechanisms of aging on longer time scales, related to the cation distribution, and the resulting changes at the atomic level have been followed through the evolution of the profiles of the x-ray diffraction peaks. 8

No attempt at quantitative interpretations of the above and similar observations are known to us, and no hint to a separation of the kinetics of the AFE and AFD OPs. It turns out that the almost ideal case of athermal transition in pure $\mathrm{PbZrO}_{3}$, amenable to a full interpretation within the Landau scheme, becomes considerably more complex with the substitution of $\mathrm{Ti}$ and other dopants, and we will limit our interpretation to the phenomenological level.

\section{B. Elastic compliance during cycling, aging and annealing}

Figure 1 1 presents a sequence of $s^{\prime}(T)$ curves measured on PZT5.4 \#1 in order to demonstrate the main features of the AFE and tilt transitions and the influence of room temperature aging and high-temperature annealing on their kinetics. Only few of the measuring cycles are shown, in order to avoid excessive crowding of the figure. Temperature was changed at rates of $\geq 1 \mathrm{~K} / \mathrm{min}$.

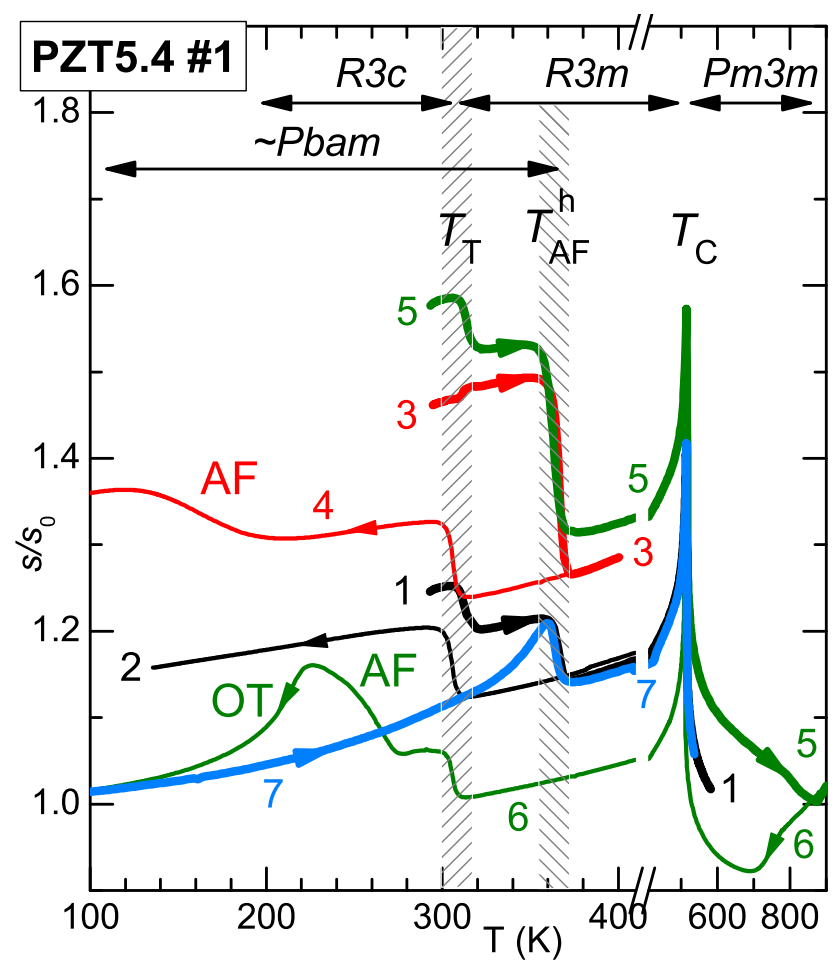

FIG. 1: Sequence of $s^{\prime}(T)$ curves of a sample of PZT5.4 with the same normalization factor $s_{0}$ corresponding to $4.2 \mathrm{kHz}$. The numbers indicate the sequence of runs; 3 was 47 days after 1 , and 5 after additional 7 days. AF and OT indicate the polar and AFD (tilt) components of the transition to the AFE-O Pbam state.

The shaded stripes mark the temperature ranges of the tilt instability in the $\mathrm{R}$ phase at $T_{\mathrm{T}}$, and the AFE to $\mathrm{FE}$ transition during heating, while the OT and AF orderings during cooling may appear at very different temperatures or may even be absent. During the first heating 
(curve 1), sharp stiffening is found both at $T_{\mathrm{T}}^{h}=314 \mathrm{~K}$ and $T_{\mathrm{AF}}^{h}=365 \mathrm{~K}$; this means that the AFE phase was only partially formed, while the rest remained $R 3 c$ and became $R 3 m$ at $T_{\mathrm{T}}^{h}$. The measurement was extended to $600 \mathrm{~K}$, well above $T_{\mathrm{C}}=514 \mathrm{~K}$ and enough to erase any memory of the AFE domains. In fact, the subsequent cooling (2) exhibits only the softening at $T_{\mathrm{T}}^{c}$ and no trace of further transitions down to $140 \mathrm{~K}$. This is the usual state of our samples with $x \simeq 0.05$, with the $\mathrm{AF} / \mathrm{OT}$ transitions very sluggish during cooling. Curve 3 was measured after 47 days, during which some measurements were done without exceeding $T_{\mathrm{C}}$. Apparently there was sufficient time for almost complete transformation into AFE/OT phase. In fact, there is only a hint of transformation at $T_{\mathrm{T}}$, and the amplitude of the step at $T_{\mathrm{AF}}^{h}$ is much enhanced, but this will be discussed more in depth in reference to Fig. 5. Part of the enhancement of the AF step, however, should be due to the fact that aging rises also the overall compliance and particularly that of the AF phase. This appears from the continuous increase with time of $s^{\prime}(400 \mathrm{~K})$, outside the stability region of the AFE phase. Curve 3 was interrupted at $400 \mathrm{~K}$, apparently before completely erasing the imprints of the AFE domains, because during cooling (4) a broad AF transition was observed at $T_{\mathrm{AF}}^{c}=165 \mathrm{~K}$. Further cycling (not shown) demonstrated that the transition to the AFE state was not complete, and also after additional 6 days curve 5 presented stiffening at $T_{\mathrm{T}}^{h}$, in addition to that at $T_{\mathrm{AF}}^{h}$. Notice that the step at $T_{\mathrm{T}}^{h}$ in curve 5 is due only to a minority fraction of R-FE phase, but its magnitude is comparable with that of curve 2, where $100 \%$ of the sample undergoes the tilt instability. This is again evidence of a general enhancement of the compliance in all phases during room temperature aging, a fact that we tentatively explain in terms of formation of extended defects that soften the lattice. Certainly a progressive softening with aging is opposite to the usual behavior of glassy systems, where aging causes a decrease of the susceptibilities.

Curve 5 was extended to $900 \mathrm{~K}$, and the subsequent cooling, 6, presents the general effect of high temperature annealing: a general restiffening to the original values and an acceleration of the kinetics of the transitions. Now both the AF softening and OT stiffening are observed during cooling, and the absence of stiffening at $T_{\mathrm{T}}$ during the subsequent heating 7 demonstrates that the transitions were complete.

\section{Extended defects}

A microscopic model of the defect structures that determine the kinetics of the AFE transition in PZT and are probably involved in the progressive softening is not available yet, but it may be related to the observations of Pokharel and Pandey ${ }^{3}$ on the AFE transition in $\mathrm{Pb}_{1-x} \mathrm{Ba}_{x} \mathrm{ZrO}_{3}$. Also in that material, the AFE/FE transformation presents huge thermal hysteresis and im- portant ageing effects over months, which have been attributed to the lattice damage generated by the large transformation strain. In particular, charged defects like dangling bonds would stabilize the FE domains over the AFE ones, making the kinetics slow. Irreversible damage like cracking and chemical decomposition certainly occurs under repeated polarization cycles and is at the origin of fatigue in capacitors and actuators, but some degree of damage may be expected to accompany even a single FE/AFE transition driven by the change of temperature, if Acoustic Emission is detected 30 Yet, there are also TEM studies indicating that there are no dislocations in correspondence with $\mathrm{AFE} / \mathrm{FE}$ boundaries, and the lattice framework is continuous. ${ }^{8}$ It has been proposed that the resulting large strain between small volume AFE and large volume FE domains promotes the short range diffusion of cations with large difference in size. ${ }^{8,31}$ Such cation exchanges require the overcoming of barriers of several eV, and therefore are practically impossible at room temperature in a homogeneous material, but the situation may be different in the highly out-ofequilibrium process of nucleation of AFE phase from the FE one. Yet, it is doubtful that such chemical inhomogeneities can be erased by simple thermal activation during short annealings at $900 \mathrm{~K}$, as in the present measurements; in fact, in the paraelectric phase there cannot be strong fields that cause out-of-equilibrium cation diffusion, and the cations should diffuse at the much higher temperatures where sintering occurs. In addition, cation diffusion has been proposed in PZT codoped with $\mathrm{La}^{3+}$ and $\mathrm{Li}^{1+}$ on the $\mathrm{Pb}^{2+}$ site, creating additional cation species widely differing in size and charge and therefore prone to rearrange, while in our case only $\mathrm{Zr}^{4+}$ and $\mathrm{Ti}^{4+}$ might undergo rearrangements.

The most obvious candidates to explain our observations of irreversible softening and recover after moderate annealing are charged $\mathrm{V}_{\mathrm{O}}$ that migrate in the internal electric fields of the FE and AFE domains and form stable structures, for example rows or even planes. This would occur mainly at twin and domain walls, $, 32,33$ where the electric and stress fields and their gradients are maximal and the interactions with the electric charges and dipoles and elastic quadrupoles of the defects are strongest. Such extended defects would in turn pin the walls and act as template for the formation of the AFE phase during subsequent transformations. That these defect structures conform to the AFE phase, during its slow formation over several days, is confirmed by the fact that the transition to the AFE phase remains easier after a brief excursion into the FE phase (curve 4 in Fig. 1). On the other hand, these extended defects must be the cause of the progressive lattice softening during aging, and are completely dissolved by annealing up to $900 \mathrm{~K}$. A brief excursion to $900 \mathrm{~K}$ in vacuum cannot heal cracks or recover a decomposed perovskite phase, and for this reason we believe that we are dealing with reversible defects like clusters of preexisting $\mathrm{V}_{\mathrm{O}}$, rather than additional loss of $\mathrm{PbO}$, cracks or cation diffusion. In order to verify that 
no appreciable loss of $\mathrm{PbO}$ or oxygen occurs during these cyclings, which are made at $2.5-4 \mathrm{~K} / \mathrm{min}$ when exceeding the Curie temperature, we repeated them in a thermobalance and found no mass loss above the background value.

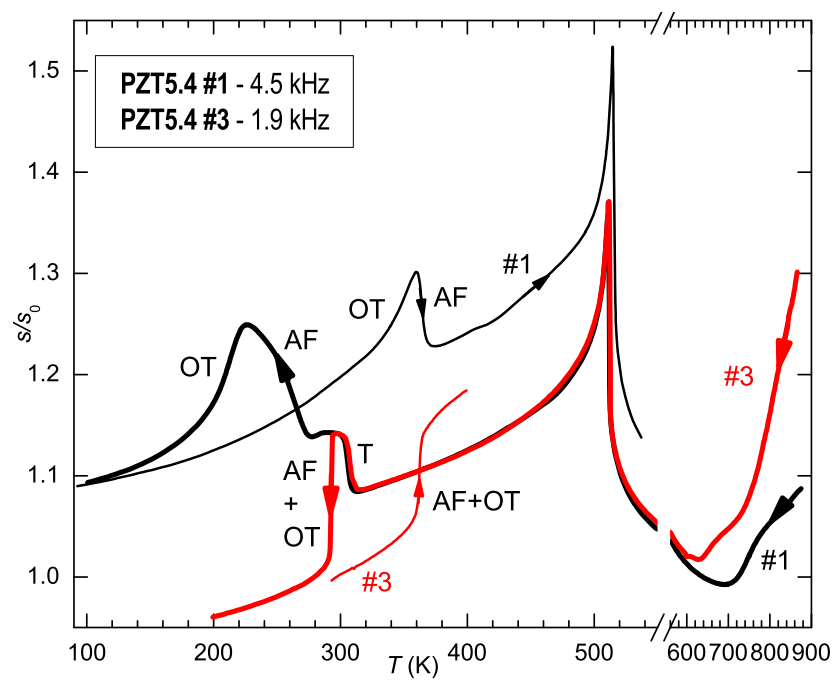

FIG. 2: Elastic compliance of two samples of PZT5.4 measured during cooling from $900 \mathrm{~K}$ (thick lines), and subsequent heating (thin lines).

These observations are in agreement with the work of Zhou et al.,$\frac{5}{5}$ where partial recovery from fatigue after $10^{8}$ strain hysteresis loops is obtained by annealing $1 \mathrm{~h}$ at $500{ }^{\circ} \mathrm{C}$. Also in that case it is concluded that migration and redistribution of charged species such as $\mathrm{O}$ vacancies must be involved, rather than healing of cracks. What is noticeable in the present experiments is that huge effects like softening of a factor of three do not occur after $10^{8}$ strain hysteresis loops, but after aging for few weeks, during which few thermal cycles are made.

Note that a quick anneal to $900 \mathrm{~K}$ recovers the original state of a sample but does not not completely erase differences from sample to sample. This is shown in Figure 2, comparing the $s^{\prime}(T)$ curves of two samples with $x=0.054$, after cycling up to $900 \mathrm{~K}$ in vacuum. The curves of sample \#1 are the same as curves 6 and 7 of Fig. 1, while the curves from sample \#3 clearly display $\mathrm{AF}$ and $\mathrm{OT}$ transitions that are very sharp and occur at exactly the same temperature both during heating and cooling. Note also the perfect reproducibility of the curves after annealing from the FE transition down to the step at $T_{\mathrm{T}}^{c}$. While Figs. 1 and 2 present cases in which the AF and OT components are sharp and coincident (PZT5.4 \#3) or OT is slower and broader than AF (PZT5.4 \#1), the third case of a slower AF component was found in samples with $5 \% \mathrm{Ti} .13$

\section{Dielectric permittivity}

In the previous work on PZT5 it was demonstrated by comparison with X-ray diffraction and dielectric spectroscopy how the softening during cooling is associated with the antipolar ordering, while the setting of the $a^{-} a^{-} c^{0}$ tilt pattern causes a stiffening. The latter could be observed as sharp steplike anomalies even when no sign of antipolar order was observed, at cooling rates $>0.5 \mathrm{~K} / \mathrm{min}$. The present samples with Ti compositions slightly above and below $5 \% \mathrm{Ti}$ display a nearly opposite behavior: during cooling at $\geq 1 \mathrm{~K} / \mathrm{min}$ the AF component is observed and starts before the OT component. We do not have an explanation for the different behaviors in the two sets of samples, prepared under very similar conditions, and checked with dielectric spectroscopy that indeed in the present samples the AF order is established also during relatively fast cooling.

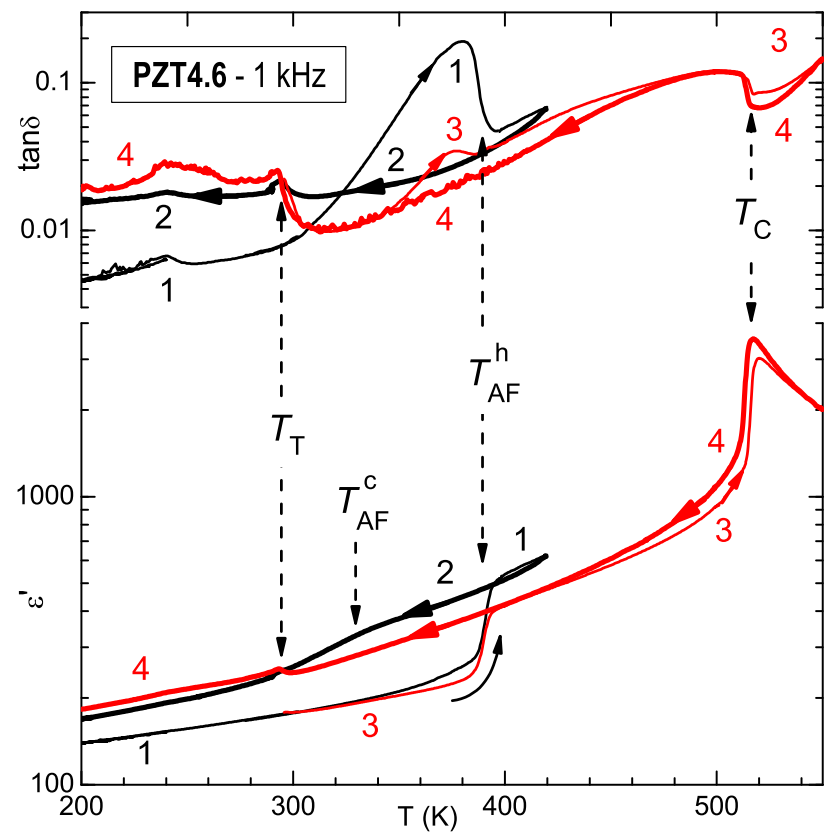

FIG. 3: Dielectric permittivity of PZT4.6 measured at $\pm 1.5 \mathrm{~K} / \mathrm{min}$. The numbers indicate the sequence of temperature runs.

Figure 3contains the dielectric spectra of PZT4.6 measured at rates of $1.5 \mathrm{~K} / \mathrm{min}$ during two cycles, in the virgin state and in the next day. Initially the sample was in the AF state and the heating curve 1 presents a clear step at $T_{\mathrm{AF}}^{h}$; the rate was inverted already at $420 \mathrm{~K}$, and the opposite step is observed at $T_{\mathrm{AF}}^{c} \simeq 330 \mathrm{~K}$ (curve 2 ). Though smaller and broader than the step at $T_{\mathrm{AF}}^{h}$, it clearly demonstrates that the AF transition indeed occurs also during cooling at $1.5 \mathrm{~K} / \mathrm{min}$ in this sample. The subsequent heating curve 3 closely follows the previous one, 1 , and was extended above $T_{\mathrm{C}}$, so erasing any memory of the AF state, and in fact no trace of AF transition is found during the cooling 4 . The anomalies in both 
real part and losses at $T_{\mathrm{T}}$ are due to the sharp octahedral tilting transition in the FE-R phase, while, as in the previous study, no signature is associated with OT tilting. The dielectric and anelastic measurements are therefore in agreement regarding the various transformation kinetics.

\section{E. Stiffening from the $\mathrm{O}$ tilt order}

The fact that stiffening rather than softening is observed at the OT transition deserves a comment. In fact, according to the established opinion, the R-FE phase of PZT is untilted above $T_{\mathrm{T}}$ and therefore tilting to the OT pattern should be accompanied by steplike softening. This derives from the Landau theory of phase transitions with linear-quadratic coupling $\lambda \varepsilon q^{2}$ between strain $\varepsilon$ and order parameter, the tilt angles $q$ of the octahedra, in this case. The resulting elastic anomaly is a negative step $\propto-\lambda^{2} / c$, where $c$ is the elastic constant of $\varepsilon, 34,35$ Other two cases have been reported where stiffening instead of the expected softening is observed at a tilt-type transition: $\mathrm{Ca}_{1-x} \mathrm{Sr}_{x} \mathrm{TiO}_{3}$ and $\mathrm{SrZrO}_{3}$ passing from a tetragonal tilted high temperature phase to the orthorhombic $a^{-} a^{-} c^{+}$tilted phase. The stiffening has been tentatively explained as due to a mutual blocking of the two order parameters having different irreducible representations, namely anti-phase tilting along $a$ and $b$ and in-phase tilting along $c \stackrel{35,36}{=}$ This explanation cannot be used for $\mathrm{PbZrO}_{3}$, whose tilt pattern $a^{-} a^{-} c^{0}$ in the $\mathrm{O}$-AFE lacks the in-phase rotation along $c$, and whose parent phase is generally considered untilted.

Our explanation 13 for stiffening in PZT is that in the R-FE phase the octahedra are tilted about all three principal cubic directions, but without long range order, so that the tilting is not easily observable by diffraction. At present there is no direct indication of disordered tilting in the $R 3 m$ phase of PZT with $x<0.06$, apart from controversia 137 observations by TEM of in-phase tilting of the $a^{0} a^{0} c^{+}$type,,$\frac{38}{r}$ and theoretical indications that the $R 3 m$ phase has strong tendency to tiltt $\stackrel{39}{ }$ Yet, the elastic compliance of PZT with $0.06 \leq x \leq 0.15$ clearly shows a transition at a temperature $T_{\mathrm{IT}}$, which can only be associated with octahedral tilting $\stackrel{18,21}{=}$ This instability line is the continuation of $T_{\mathrm{T}}(x)$ when it starts dropping below $x \sim 0.15$ and merges with $T_{\mathrm{C}}$ at $x=0.06$. It follows that, when $x<0.06$, the $\mathrm{FE}$ transition at $T_{\mathrm{C}}$ also involves disordered tilting $\underset{21}{\underline{21}}$ Then, the OT transition to the $a^{-} a^{-} c^{0}$ pattern occurs through the loss of tilting about the $c$ axis, and may well cause stiffening.

\section{F. Fits of elastic anomalies}

In what follows we present several pairs of successive heating and cooling cycles with the corresponding curves of the compliance and of the losses, extending the range of shapes of the elastic anomalies with respect to the previous work on PZT5 $\stackrel{13}{=}$ In all cases it is possible to fit the curves with the hypothesis that the compliance $s$ is the average of the compliances $s_{k}$ of the coexisting phases, weighted with their volume fractions $f_{k}$ :

$$
s=\sum_{k} f_{k} s_{k}
$$

with $k=\mathrm{FE}$ (untilted R-FE $R 3 m$ ), $\mathrm{T}\left(a^{-} a^{-} a^{-}\right.$tilting of the R-FE $R 3 c$ phase), AFE (only cation displacements without long range $a^{-} a^{-} c^{0}$ tilt order) and OT (long range $a^{-} a^{-} c^{0}$ tilt order). The reference compliance is $s_{\mathrm{FE}}$ of the $R 3 \mathrm{~m}$ phase, assumed to include the anharmonic dependence on temperature; the latter is taken as linear. Notice that some phases are mutually exclusive and some are not: the FE and AFE cation orderings and the T $\left(a^{-} a^{-} a^{-}\right)$and OT $\left(a^{-} a^{-} c^{0}\right)$ tilt patterns are exclusive pairs, but $\mathrm{T}$ may in principle persist within AFE domains. These conditions are reflected in the volume fractions. In some cases it is found necessary to introduce an interaction between the AFD and polar orderings, with the assumption that the OT tilting occurs only within $\mathrm{AFE}$ domains. If the volume fractions change sharply between 0 and 1 at the transition temperatures, one gets steplike changes of the elastic constant at the transitions, as expected from the Landau theory for phase transitions with order parameters whose square is linearly coupled to strain $; 4,35$ this is indeed the case of the AFD and polar transitions. The steplike elastic anomaly is combined with the condition that each transition occurs over a certain time and temperature range, setting the volume fractions $f_{k}$ as steps centered at the transition temperatures $T_{k}$ with widths $\Delta T_{k}$,

$$
f_{k}=\frac{1}{2}\left[1-\tanh \left(\frac{T-T_{k}}{\Delta T_{k}}\right)\right] .
$$

The variety of the shapes of the anomalies derives from the strong dependence of $T_{k}$ and $\Delta T_{k}$ on the sample state and temperature rate. Certainly expressions (1) is a simplification, especially when describing transitions over broad temperature ranges and measured at a variable temperature rate, but it will be shown that even with this approximation remarkably good fits are possible. When the OT pattern sets in, it replaces the T pattern, and therefore $f_{\mathrm{T}}$ has to be multiplied by $1-f_{\mathrm{OT}}$. The fitting formula becomes

$$
s=b_{0}+b_{1} T+f_{\mathrm{T}}\left(1-f_{\mathrm{OT}}\right) A_{\mathrm{T}}+f_{\mathrm{OT}} A_{\mathrm{OT}}+f_{\mathrm{AF}} A_{\mathrm{AF}}
$$

where $A_{k}=s_{k}-s_{\mathrm{FE}}$ are the amplitudes of the steps. In certain cases it appears that the OT pattern does not sets in independently of the AFE or FE order, but only after the occurrence of the AFE transition. In these cases the OT contribution is set as $f_{\mathrm{OT}} f_{\mathrm{AF}} A_{\mathrm{OT}}$. In many cases it is clear that the transition to the AFE or OT state is incomplete during cooling, and in these cases the corresponding volume fraction is let to increase up to $f_{\mathrm{AF}}<1$. 
In the following series of figures, the temperature dependences of the compliance and of the losses are presented in pairs of successive heating and cooling cycles with rates of $\sim 1 \mathrm{~K} / \mathrm{min}$ unless otherwise specified. The continuous lines are fits to the real parts, while no attempt has been done to fit the losses. The latter are in some case noisy and therefore the $s^{\prime \prime} / s^{\prime}$ curves are splines passing through the data points. The vertical arrows indicate the transition temperatures $T_{k}$, while the error bars extend between $T_{k} \pm \Delta T_{k}$.

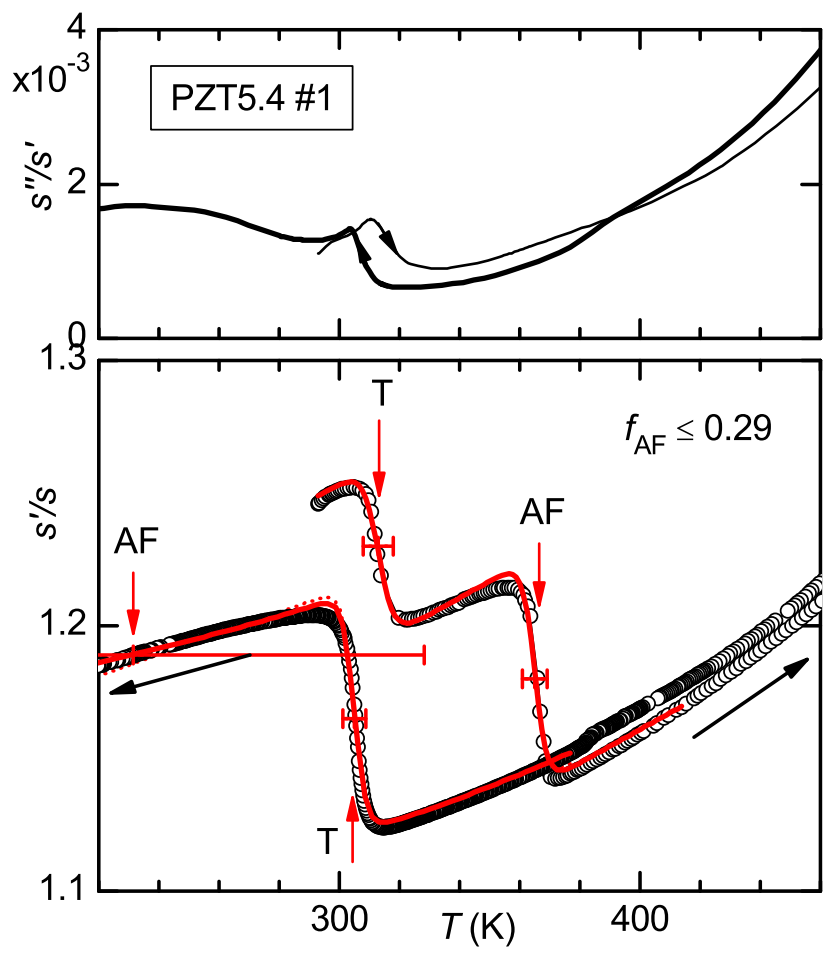

FIG. 4: (Color online) Elastic compliance of PZT5.4 \#1 during a heating/cooling cycle in the virgin state. The continuous lines of $s^{\prime \prime} / s^{\prime}$ are splines through the experimental points; the experimental points of $s^{\prime} / s_{0}$ (curves 1 and 2 of Fig. 1) are fitted with the continuous lines. The vertical arrows indicate the fitting values $T_{k}$ of the steps and the error bars their widths $\pm \Delta T_{k}$; the fraction of AFE phase was limited to $f_{\mathrm{AF}} \leq 0.29$ instead of 1 . Heating and cooling rates of approximately $1 \mathrm{~K} / \mathrm{min}$.

We start with the first heat/cool cycle on sample PZT5.4 \#1 (Fig. 4 and curves 1 and 2 of Fig. 11.). In this initial state a partial transformation to the AFE state had occurred, and hence both the stiffening at $T_{\mathrm{T}}^{h}=313 \mathrm{~K}$ from the untransformed R-FE phase and the stiffening at $T_{\mathrm{AF}}^{h}=365 \mathrm{~K}$ are present. The ramp was extended up to $600 \mathrm{~K}$ (well above $T_{\mathrm{C}}=513 \mathrm{~K}$ ), therefore erasing the memory of the AFE domains, and no evidence is found of AFE or OT transformation during the subsequent cooling. The initial AFE fraction $f_{\mathrm{AF}}=0.29$ can be deduced from the ratio of the initial step at $T_{\mathrm{T}}^{h}$ to that at $T_{\mathrm{T}}^{c}$, which is equal to $1-f_{\mathrm{AF}}$. A slight improvement of the fit for the cooling curve is obtained assuming a broad AF transition centered at $T_{\mathrm{AF}}^{c} \simeq 230 \mathrm{~K}$, whose high temperature tail improves the shape of the step.

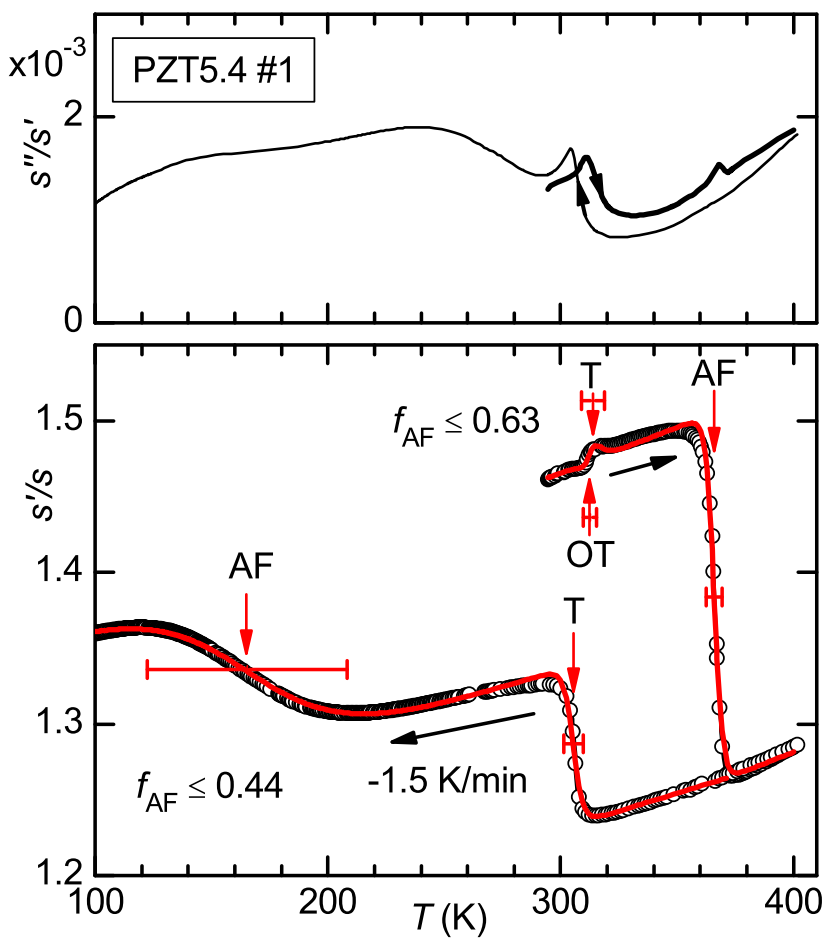

FIG. 5: (Color online) Elastic compliance of PZT 5.4 \#1 measured 47 days after Fig. 4

The next curves (Fig. 5 corresponding to curves 3 and 4 of Fig. 1) are measured after 47 days, during which almost complete transformation to the $\mathrm{AF}+\mathrm{OT}$ phase should have occurred, judging from the seeming lack of the negative step at $T_{\mathrm{T}}^{h}=313 \mathrm{~K}$ (see e.g. Fig. 4). In its place, a small positive step with a wavelet is found, which suggests the mutual cancelation of OT and T steps at nearly the same temperature but with different widths. In most cases, the OT and AF transitions occur together during heating (Ref. 13, curve \#1 in Fig. 2, Figs. 9 and 10), but in sample PZT5.4 \#1 the OT tilting appears less stable, and it seems that in Fig. 5 the disappearance of $a^{-} a^{-} c^{0}$ tilting is triggered by the disappearance of $a^{-} a^{-} a^{-}$tilting in the neighboring R-FE domains at $T_{\mathrm{T}}^{h}$, before the transition to the FE state occurs. If one assumes that the OT component disappears completely at $T_{\mathrm{OT}}^{h} \simeq T_{\mathrm{T}}^{h}$, there is uncertainty on the magnitude of the mutually canceled $\mathrm{T}$ and $\mathrm{OT}$ steps, which reflects in an uncertainty in the fraction $f_{\mathrm{AF}}$ of AFE phase in the initial state. The fit of Fig. 5 is done setting $A_{\mathrm{OT}}=$ 0.1 , similarly to other cases, which results in $f_{\mathrm{AF}} \simeq 0.63$ during heating and 0.44 during cooling, but equivalent fits can be obtained by setting a smaller $A_{\mathrm{OT}}$, with the initial $f_{\mathrm{AF}}$ up to 1 . During cooling no sign of transformation to the OT phase is found. As before, the losses present a steplike increase in the tilted $\mathrm{R}$ phase, $T<T_{\mathrm{T}}$, and an anomaly is hardly visible at $T_{\mathrm{AF}}^{h}$.

Figure 6 corresponds to curves 5 and 6 of Fig. 11 mea- 


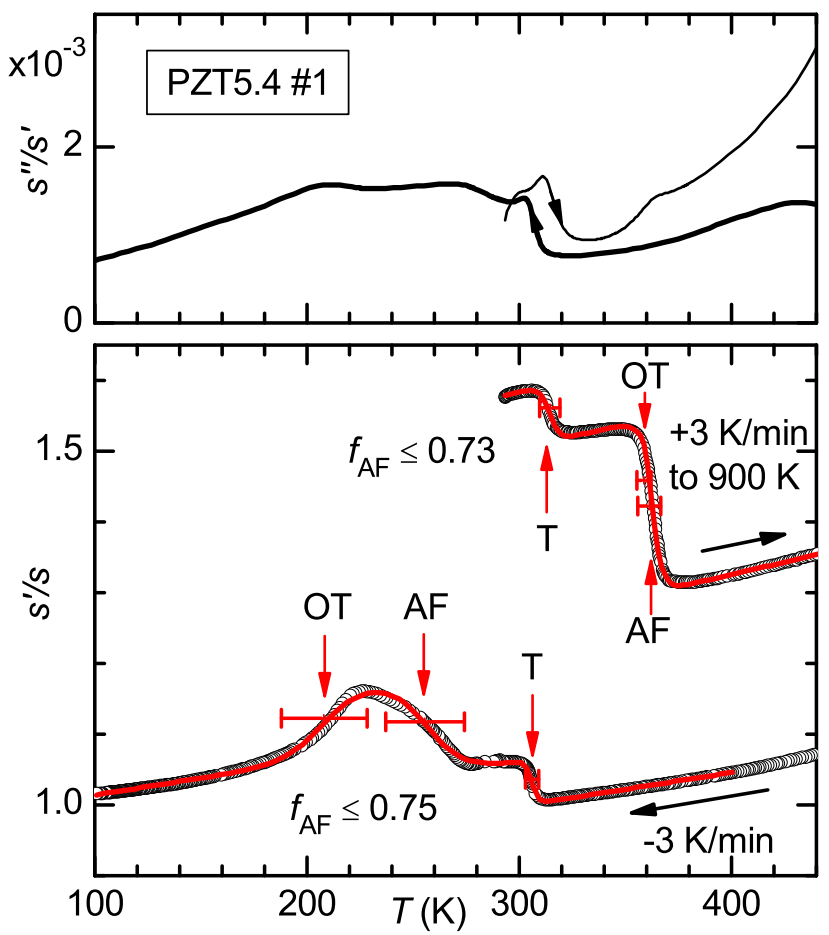

FIG. 6: (Color online) Curves 5 and 6 of Fig. 1 including heating up to $900 \mathrm{~K}$.

sured at $\pm 3 \mathrm{~K} / \mathrm{min} ; 6$ days after the previous run, a considerable fraction of $\mathrm{AF}+\mathrm{OT}$ phase had formed, but there is ambiguity on how much the two transitions contribute to the step at $T_{\mathrm{AF}}^{h}=361.3 \mathrm{~K} \simeq T_{\mathrm{OT}}^{h}$. In fact, the heating was extended to $900 \mathrm{~K}$, causing a recover of the overall compliance and of the compliance steps to the original lower values. Therefore, it was not possible to share in the fit the amplitudes of the steps between heating and cooling, as in all other cases. We chose to share only $A_{\mathrm{OT}}$. Notice that, thanks to the dissolution at $900 \mathrm{~K}$ of the defects that stabilized the FE phase, both the AF and OT transitions are observed even at a cooling rate as fast as $3 \mathrm{~K} / \mathrm{min}$. It is possible to obtain very similar fits of the broad split AF+OT transition both with and without the condition that the OT is subordinated to $\mathrm{AF}$; the only parameters that are affected by the choice are the step amplitudes $A_{\mathrm{AF}}$ and $A_{\mathrm{OT}}$, which change by less than $10 \%$.

The final fit of the PZT5.4 \#1 sample (Fig. 7) is a combination of the previous cooling in the annealed state and the subsequent heating. Also in this case it was impossible to share the amplitude $A_{\mathrm{AF}}$ between cooling and heating, because its magnitude is clearly greater during cooling. In this case the heating curve clearly indicates that the OT order is subordinated to the AF order. In fact, the slow upturn during heating is fitted with a broad OT anomaly, centered at a temperature $T_{\mathrm{OT}}^{h}$ very close to $T_{\mathrm{AF}}^{h}$, as usually is the case during heating, but the slow upturn would continue also above $T_{\mathrm{AF}}^{h}$ as in the dashed curve. The maximum fraction of transformed AFE phase

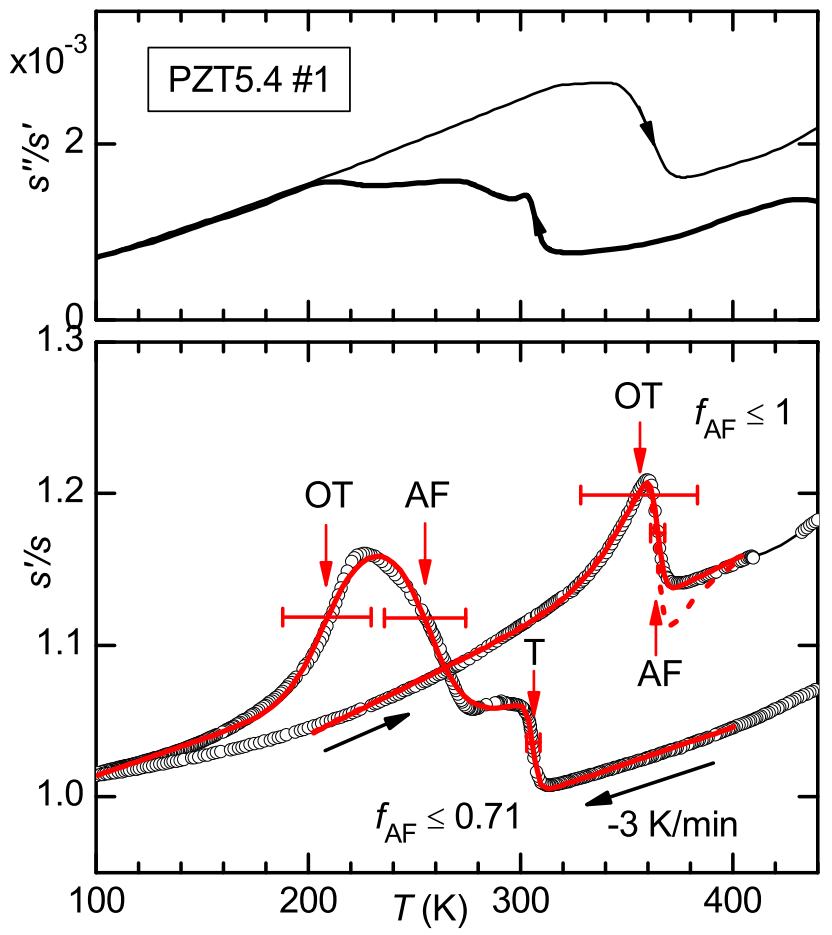

FIG. 7: (Color online) Curves 6 and 7 of Fig. 1 The dashed line is obtained assuming that AF and OT are independent of each other.

on cooling was not imposed and left as a free parameter, but its value 0.71 is consistent with that of the previous fit $\left(f_{\mathrm{AF}}=0.76\right)$. These curves demonstrate that some interaction between the polar and AFD modes exists, and it is not always possible to fit elastic anomalies overlapping over several tens of kelvin as if they were completely independent of each other.

We pass with Fig. 8 to PZT4.6 \#1 in its virgin state. At this composition $T_{\mathrm{T}} \simeq T_{\mathrm{AF}}^{c, 0} \simeq$ room temperature, but from the lack of stiffening at $T_{\mathrm{T}}^{h}$ it is deduced that the sample was initially completely transformed in the $\mathrm{AF}+\mathrm{OT}$ state and reverted sharply to the untilted FE phase. The combination of stiffening $(\mathrm{AF} \rightarrow \mathrm{FE})$ and softening (loss of long range OT tilt order) at practically the same temperature produces a spike in the compliance. The heating was extended to $T_{\mathrm{C}}+50 \mathrm{~K}=560 \mathrm{~K}$, but contrary to the PZT5.4 and also PZT5 cases, both the AF and OT transitions were complete, though broadened, also during cooling. All the previous fits to the PZT5.4 curves were made imposing that OT tilting takes place only within AF domains (see especially Fig. 17), but the fit to the virgin PZT4.6 \#1 is much better by relaxing this condition. This was also the case of PZT5, where sharp OT ordering occurred without observable formation of AF order.

After 25 days of aging the compliance at room temperature had increased of more than three times (Fig. 9), which is really an enormous effect, with a softening mainly developed in the AF phase, but also of the $\mathrm{FE}$ 


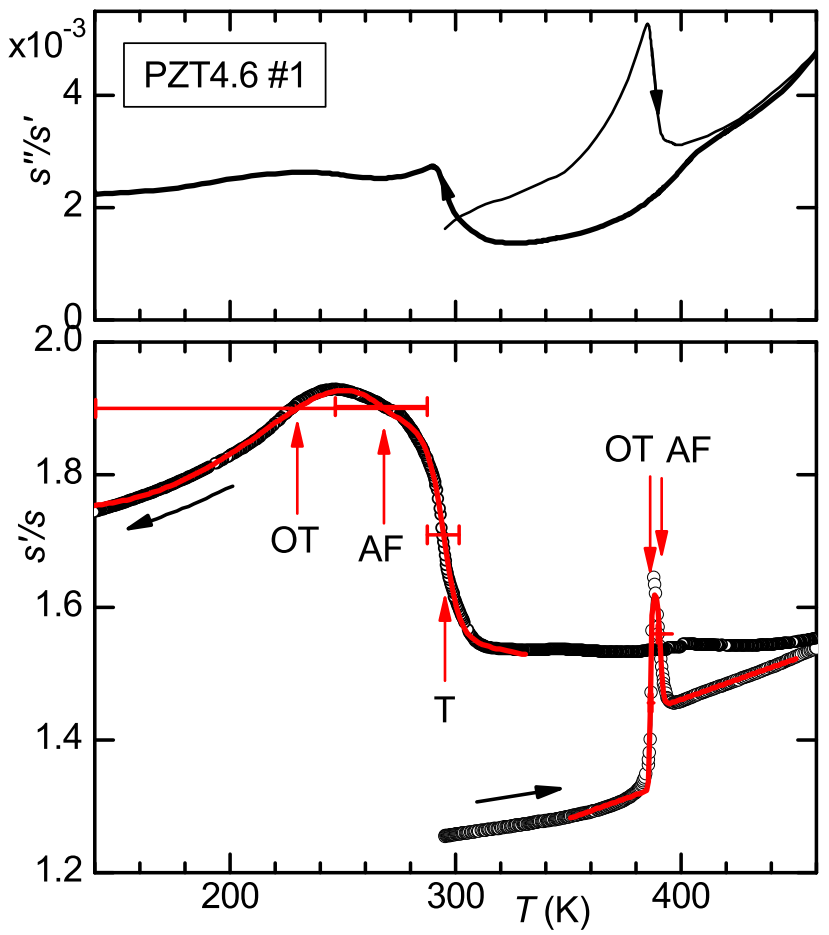

FIG. 8: (Color online) Anelastic spectrum of PZT4.6 \#1 during a heating/cooling cycle in is virgin state.

phase became 1.7 times softer than initially. The run was extended to $625 \mathrm{~K}$ and produced a partial anneal of the defects and recovery of the stiffness. During cooling, the $\mathrm{AF}$ and $\mathrm{OT}$ transitions were visible but broadened and possibly only partial: the fit was done by sharing the amplitudes of the steps but reducing the fraction $f_{\mathrm{AF}} \leq 0.55$ during cooling, but it is also possible that the amplitudes of the steps were halved by the partial annealing and the fraction of transformed phase was $f_{\mathrm{AF}} \simeq 1$. As discussed in reference to Fig. 7 the broad OT transition on heating had to be forced to follow the sharper AF one, in order to avoid an undershoot at $T>T_{\mathrm{AF}}^{h}$.

The last pair of fits (Fig. 10) is from another sample, PZT4.6 \#3, measured after high-temperature annealing. The amplitudes of the AF and OT steps are very close to those of the virgin PZT4.6 \#1, the main difference being that virgin PZT4. 6 \#1 had broad transitions during cooling, while annealed PZT4.6 \#3 has a cooling curve similar to the heating one, with sharp and almost coincident $\mathrm{AF}$ and $\mathrm{OT}$ transitions.

\section{CONCLUSIONS}

The phase diagram of PZT near the boundary between $\mathrm{FE}$ and AFE phases is the result of the action of polar, antipolar and octahedral tilt instabilities, but also by yet scarcely characterized defects. Such defects, which we tentatively identify with variously aggregated $\mathrm{O}$ vacan-

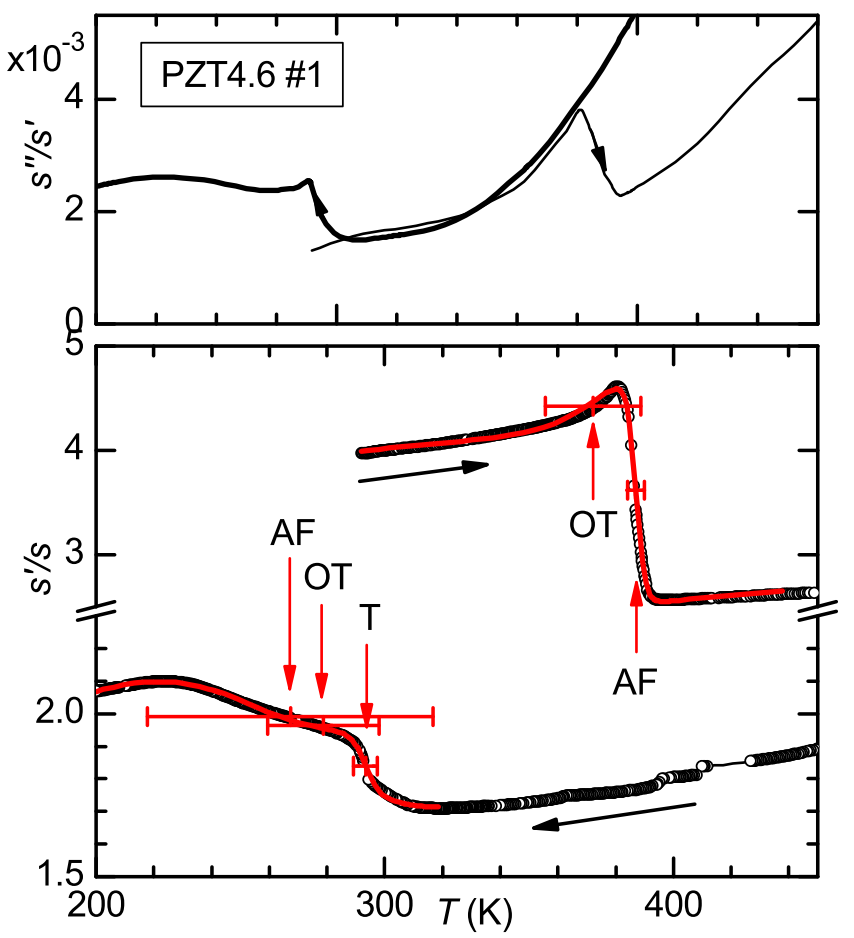

FIG. 9: (Color online) Anelastic spectrum of PZT4.6 \#1 25 days after the measurement of Fig. 8. The cycle was extended to $900 \mathrm{~K}$

cies, can drastically change the kinetics of the different modes involved in the transition to the AFE state, causing a freezing of the tilt or antipolar modes or both, but also more than doubling in a reversible way the elastic compliance. This is in marked contrast with the aging usually found in glassy and disordered systems, but also FE or relaxor PZT $\stackrel{40.41}{\longleftarrow}$ which is accompanied by a decrease of the susceptibility or stiffening.

As a result of the interplay of the different polar and tilt modes and probably defect aggregation, the curves of the elastic compliance versus temperature exhibit a variety of shapes, which however can be well fitted with simple hypotheses for the studied compositions $0.046 \leq$ $x \leq 0.054$. There are three transitions connected with the establishment of 1) polar/antipolar order, 2) octahedral tilt pattern in the AFE orthorhombic phase $\left(a^{-} a^{-} c^{0}\right.$, here labeled $\left.\mathrm{OT}\right)$, and 3$)$ tilt pattern in the $\mathrm{FE}$ rhombohedral phase $\left(a^{-} a^{-} a^{-}\right)$. Each transition causes a steplike change in the elastic compliance, whose temperature and width depend on the kinetics of the transition. These steps can be well approximated with hyperbolic tangents of $T$, whether sharp or broad, and the imperfect fits are probably due to variations in the temperature rates as much as to the choice of the hyperbolic tangent as fitting function.

In most of the presently studied samples the octahedral tilt order OT seems less stable and subordinated to the AFE one, but there are cases where the fit requires that the two are independent (Fig. 8), and this is espe- 


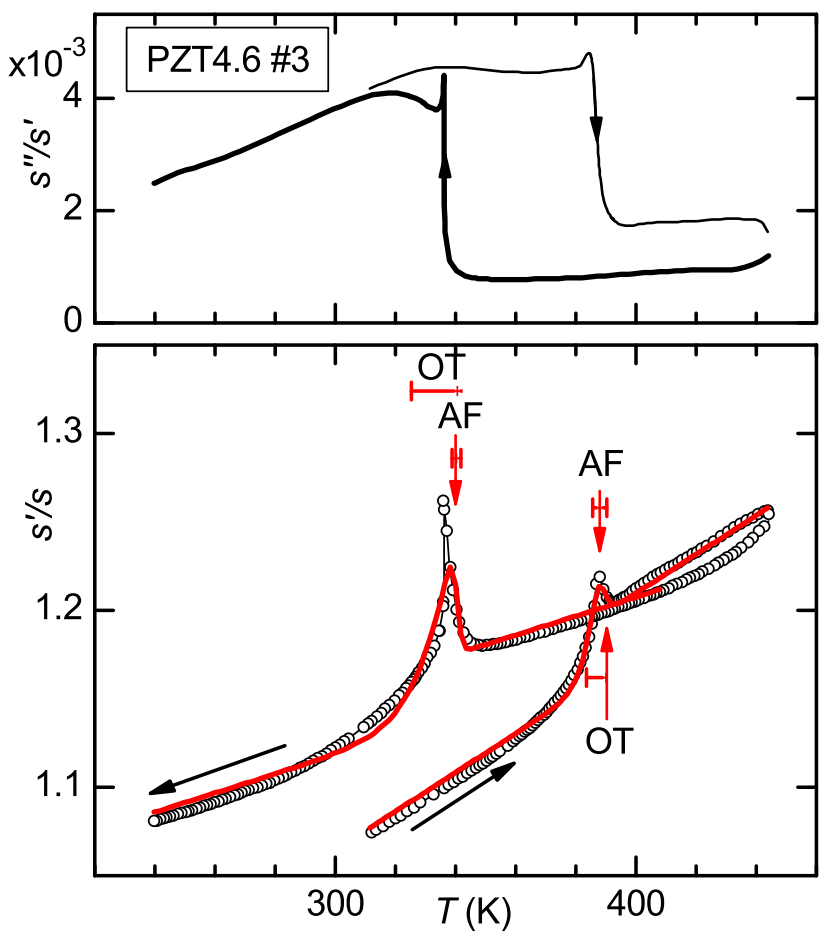

FIG. 10: (Color online) Anelastic spectrum of PZT4.6 \#3 5 days after annealing up to $860 \mathrm{~K}$.

cially true in the previous study on PZT $5, \frac{13}{1}$ where sharp
OT transitions occur in the absence of the AFE ones. A case is also presented (Fig. 51) where the loss of OT tilting appears to be triggered by the loss of tilting in the coexisting R-FE domains.

The slow kinetics for the onset of the $\mathrm{AF}+\mathrm{OT}$ order is due to some type of aggregation of mobile defects, which causes a stabilization of the FE phase and a general softening of the lattice, by factors as large as 2.3 within the FE phase and even 4 at room temperature, where in addition the state can vary between $\mathrm{FE}$ and $\mathrm{AFE}$. We emphasize that such macroscopic changes of the elastic properties occur simply during room temperature aging or after few $\mathrm{FE} / \mathrm{AFE}$ cycles, and not after repeated electric field induced cycling as in fatigue experiments. Moderate annealing at $900 \mathrm{~K}$, well below the sintering temperature, can recover the pristine elastic stiffness and promptness to form the AFE phase. This fact suggests that the defects involved are $\mathrm{O}$ vacancies rather than cracks, chemical decomposition or cation diffusion, which instead are relevant in fatigue experiments.

\section{Acknowledgments}

The authors thank C. Capiani (ISTEC) for the skillful preparation of the samples, P.M. Latino (ISC) and R. Scaccia (ISC) for their technical assistance in the anelastic and dielectric experiments.
1 K. Uchino, Acta Mater. 46, 3745-3753 (1998).

${ }^{2}$ X. Hao, J. Zhai, L.B. Kong and Z. Xu, Progr. Mater. Sci. 63, 1 (2014).

3 B.P. Pokharel and D. Pandey, J. Appl. Phys. 86, 3327 (1999).

4 B.P. Pokharel and D. Pandey, J. Mater. Sci. 42, 10239 (2007).

5 L. Zhou, A. Zimmermann, Y.-P. Zeng and F. Aldinger, J. Am. Ceram. Soc. 87, 1591 (2004).

6 X.J. Lou, J. Appl. Phys. 105, 024101 (2009).

7 V.M. Ishchuk and V.L. Sobolev, Ferroelectrics 413, 409 (2011).

8 V.M. Ishchuk, Z.A. Samoylenko and V.L. Sobolev, J. Phys.: Condens. Matter 18, 11371 (2006).

9 V.M. Ishchuk, Ferroelectrics 255, 73 (2001).

${ }^{10}$ K.Z. Baba-Kishi and D.J. Barber, J. Appl. Cryst. 23, 43 (1990).

11 I.M. Reaney, D.I. Woodward and C.A. Randall, J. Am. Ceram. Soc. 94, 2242 (2011).

12 S. Watanabe and Y. Koyama, Phys. Rev. B 63, 134103 (2001).

13 F. Cordero, F. Craciun, F. Trequattrini, C. Galassi, P.A. Thomas, D.S. Keeble and A.M. Glazer, Phys. Rev. B 88, 094107 (2013).

14 A.M. Glazer, Acta Cryst. B 28, 3384 (1972).

15 D. Wang, E.K.H. Salje, S.-B. Mi, C.-L. Jia and L. Bellaiche, Phys. Rev. B 88, 134107 (2013).
16 J.-W. Kim, P. Thompson, S. Brown, P.S. Normile, J.A. Schlueter, A. Shkabko, A. Weidenkaff and P.J. Ryan, Phys. Rev. Lett. 110, 027201 (2013).

17 F. Cordero, F. Craciun and C. Galassi, Phys. Rev. Lett. 98, 255701 (2007).

18 F. Cordero, F. Trequattrini, F. Craciun and C. Galassi, J. Phys.: Condens. Matter 23, 415901 (2011).

19 F. Cordero, L. Dalla Bella, F. Corvasce, P.M. Latino and A. Morbidini, Meas. Sci. Technol. 20, 015702 (2009).

20 A.S. Nowick and B.S. Berry, Anelastic Relaxation in Crystalline Solids. (Academic Press, New York, 1972).

21 F. Cordero, F. Trequattrini, F. Craciun and C. Galassi, Phys. Rev. B 87, 094108 (2013).

${ }^{22}$ H. Fujishita and S. Hoshino, J. Phys. Soc. Japan 53, 226 (1984).

23 A.M. Glazer, K. Roleder and J. Dec, Acta Cryst. B 49, 846 (1993).

${ }^{24}$ H. Fujishita, Y. Ishikawa, S. Tanaka, A. Ogawaguchi and S. Katano, J. Phys. Soc. Japan 72, 1426 (2003).

25 J. Handerek and Z. Ujma, J. Phys.: Condens. Matter 7, 1721 (1995).

26 S. Kojima, N. Ohta and X. Dong, Jpn. J. Appl. Phys. 38, 5674 (1999).

27 D. Viehland, J.-F. Li, X. Da and Z. Xu, J. Phys. Chem. Sol. 57, 1545 (1996).

28 S. Watanabe and Y. Koyama, Phys. Rev. B 66, 134102 (2002). 
29 J. Dec, K. Roleder and K. Stroi, Solid State Commun. 99, 157 (1996).

30 E. Dulkin, B. Mihailova, G. Catalan, M. Gospodinov and M. Roth, Phys. Rev. B 82, 180101 (2010).

31 V.M. Ishchuk and V.L. Sobolev, J. Appl. Phys. 92, 2086 (2002).

32 Q. Tan, Z. Xu, J.-F. Li and D. Viehland, Appl. Phys. Lett. 71, 1062 (1997).

33 W.T. Lee, E.K.H. Salje and U. Bismayer, Phys. Rev. B 72, 104116 (2005).

34 W. Rehwald, Adv. Phys. 22, 721 (1973).

35 R.A. McKnight, C.J. Howard and M.A. Carpenter, J. Phys.: Condens. Matter 21, 015901 (2009).
36 Z. Zhang, J. Koppensteiner, W. Schranz, J.B. Betts, A. Migliori and M.A. Carpenter, Phys. Rev. B 82, 014113 (2010).

37 D.I. Woodward, J. Knudsen and I.M. Reaney, Phys. Rev. B 72, 104110 (2005).

38 D. Viehland, Phys. Rev. B 52, 778 (1995).

39 K. Leung, E. Cockayne and A.F. Wright, Phys. Rev. B 65, 214111 (2002).

40 F. Cordero, F. Craciun, A. Franco, D. Piazza and C. Galassi, Phys. Rev. Lett. 93, 097601 (2004).

41 F. Cordero, F. Craciun, A. Franco and C. Galassi, Phys. Rev. B 74, 024110 (2006). 